\title{
Superparamagnetic Nanoparticle Clusters for Cancer Theranostics Combining Magnetic Resonance Imaging and Hyperthermia Treatment
}

\author{
Koichiro Hayashi ${ }^{\bowtie}$, Michihiro Nakamura ${ }^{1}$, Wataru Sakamoto², Toshinobu Yogo'2, Hirokazu Miki³, Shuji \\ Ozaki $^{4}$, Masahiro Abe ${ }^{5}$, Toshio Matsumoto ${ }^{5}$, and Kazunori Ishimura ${ }^{1}$ \\ 1. Department of Anatomy and Cell Biology, Institute of Health Biosciences, The University of Tokushima Graduate School, 3-18-15 Ku- \\ ramoto-cho, Tokushima, 770-8503, Japan; \\ 2. Division of Nanomaterials Science, EcoTopia Science Institute, Nagoya University, Furo-cho, Chikusa-ku, Nagoya 464-8603, Japan; \\ 3. Division of Transfusion Medicine, Tokushima University Hospital 2-50-1 Kuramoto-cho, Tokushima, 770-0042, Japan; \\ 4. Department of Internal Medicine, Tokushima Prefectural Central Hospital, 1-10-3 Kuramoto-cho, Tokushima, 770-0042, Japan; \\ 5. Department of Medicine and Bioregulatory Sciences, The University of Tokushima Graduate School of Medical Sciences, 3-18-15 Ku- \\ ramoto-cho, Tokushima, 770-8503, Japan. \\ $\square$ Corresponding author: Koichiro Hayashi, Ph.D. hayashi@tokushima-u.ac.jp. Phone: (088) 633 9220. Fax: (088) 6339426.
}

( ) Ivyspring International Publisher. This is an open-access article distributed under the terms of the Creative Commons License (http://creativecommons.org/ licenses/by-nc-nd/3.0/). Reproduction is permitted for personal, noncommercial use, provided that the article is in whole, unmodified, and properly cited.

Received: 2013.01.10; Accepted: 2013.02.27; Published: 2013.04.23

\begin{abstract}
Superparamagnetic nanoparticles (SPIONs) could enable cancer theranostics if magnetic resonance imaging (MRI) and magnetic hyperthermia treatment (MHT) were combined. However, the particle size of SPIONs is smaller than the pores of fenestrated capillaries in normal tissues because superparamagnetism is expressed only at a particle size $<10 \mathrm{~nm}$. Therefore, SPIONs leak from the capillaries of normal tissues, resulting in low accumulation in tumors. Furthermore, MHT studies have been conducted in an impractical way: direct injection of magnetic materials into tumor and application of hazardous alternating current (AC) magnetic fields. To accomplish effective enhancement of MRI contrast agents in tumors and inhibition of tumor growth by MHT with intravenous injection and a safe AC magnetic field, we clustered SPIONs not only to prevent their leakage from fenestrated capillaries in normal tissues, but also for increasing their relaxivity and the specific absorption rate. We modified the clusters with folic acid (FA) and polyethylene glycol (PEG) to promote their accumulation in tumors. SPION clustering and cluster modification with FA and PEG were achieved simultaneously via the thiol-ene click reaction. Twenty-four hours after intravenous injection of FA- and PEG-modified SPION nanoclusters (FA-PEG-SPION NCs), they accumulated locally in cancer (not necrotic) tissues within the tumor and enhanced the MRI contrast. Furthermore, $24 \mathrm{~h}$ after intravenous injection of the $\mathrm{NCs}$, the mice were placed in an $\mathrm{AC}$ magnetic field with $H=8 \mathrm{kA} / \mathrm{m}$ and $f=230 \mathrm{kHz}\left(H f=1.8 \times 10^{9} \mathrm{~A} / \mathrm{m} \cdot \mathrm{s}\right)$ for $20 \mathrm{~min}$. The tumors of the mice underwent local heating by application of an $A C$ magnetic field. The temperature of the tumor was higher than the surrounding tissues by $\approx 6^{\circ} \mathrm{C}$ at $20 \mathrm{~min}$ after treatment. Thirty-five days after treatment, the tumor volume of treated mice was one-tenth that of the control mice. Furthermore, the treated mice were alive after 12 weeks; control mice died up to 8 weeks after treatment.
\end{abstract}

Key words: theranostics, nanomedicine, iron oxide, MRI, hyperthermia, tumor.

\section{INTRODUCTION}

"Theranostics" is a combination of diagnostic and local treatment of lesions.[1-5] It has the potential to change the current medical paradigm of "see and treat" to "detect and inhibit". Magnetic nanoparticles 
can potentially achieve the theranostics combining magnetic resonance imaging (MRI) and magnetic hyperthermia treatment (MHT) because they not only shorten the transverse relaxation time $\left(T_{2}\right)$ of protons and lead to a darkening in the reconstructed image, but also generate heat by the application of alternating current (AC) magnetic fields.[6-9]

MRI is a diagnostic imaging modality and provides anatomical information. ${ }^{[10-13]}$ Superparamagnetic iron oxide nanoparticles (SPIONs) are used as negative contrast agents for MRI, but their application is limited mainly to the detection of liver cancer. Therefore, MRI contrast agents for the detection of non-liver cancer have been studied. In the development of MRI contrast agents, ensuring safety is the primary issue. In this regard, SPION-based materials are promising as MRI contrast agents because SPIONs are already used in clinical practice and are believed to be non-toxic.

MRI contrast agents are also required to have high relaxivity $\left(r_{2}=T_{2}{ }^{-1}\right)$ for shortening the $T_{2}$ effectively. Experiments have shown that the clustering of SPIONs is an effective approach for increasing $r_{2}$ dramatically.[14-16] Moreover, cancer tissues and necrotic tissues are distributed heterogeneously within the tumor. Therefore, confirmation as to whether MRI contrast agents are located in cancer tissues should be sought.

MHT is a promising cancer thermotherapy because magnetic materials produce heat under AC magnetic fields. ${ }^{[17]}$ MHT enables the local treatment of tumors without damage to normal tissue because tumors are not only susceptible to heat but are also readily heated compared with normal tissue. The number of surviving cancer cells decrease gradually below $42^{\circ} \mathrm{C}$, but decrease rapidly above $42^{\circ} \mathrm{C}$. Numerous in vitro studies on MHT have been reported, but few in vivo studies gave been conducted. ${ }^{[18-20]}$ In vivo studies on MHT have hitherto been carried out by direct injection of magnetic nanoparticles into the tumor, which is not particularly practical. MHT involving intravenous injection of magnetic nanoparticles has practical advantages. Furthermore, this type of MHT can be carried out directly after MRI.

Most of the studies on MHT used harmful AC magnetic fields for patients. Experiments have shown that the product of the amplitude $(H)$ and frequency (f) of a magnetic field should be $<5 \times 10^{9} \mathrm{Am}^{-1} \mathrm{~s}^{-1}$. [21,22] Therefore, data on the therapeutic effect of MHT assessed under AC magnetic fields with $\mathrm{H} f$ of $<5 \times 10^{9}$ $\mathrm{Am}^{-1} \mathrm{~s}^{-1}$ is required. We have revealed that SPIONs are more suitable than ferromagnetic magnetic nanoparticles as heating elements for MHT. This is because the amount of heat generated by SPIONs is readily controllable by tuning $H$ and $f$ outside the body, ${ }^{[23,24]}$ whereas that of ferromagnetic magnetic nanoparticles is difficult to control because it is largely dependent upon particle size and is markedly decreased at $>16 \mathrm{~nm} .{ }^{[25]}$ However, superparamagnetism is expressed only at a particle size $<10 \mathrm{~nm}$, so SPIONs "leak" from the pores of fenestrated capillaries in normal tissues: the pores are a few dozen nanometers and are smaller than the pores of the vascular walls in tumors. Hence, SPIONs do not accumulate in tumors. However, the clustering of SPIONs can be a way out of this dilemma. SPION clustering increases the particle size up to the optimum size for making use of the enhanced permeability and retention (EPR) effect,[26] while maintaining their superparamagnetic properties.

The effective delivery of nanoparticles to tumors is absolutely imperative for theranostic systems combining MRI and MHT. The surface modification of nanoparticles with polyethylene glycol (PEG), known as PEGylation, inhibits phagocytosis by the reticuloendothelial system (RES), and prolongs the retention time in blood; these factors promote the EPR effect.[27] Folic acid (FA) binds to folate receptors, which are overexpressed in various types of human cancer cells but which, in general, are absent in most normal cells. ${ }^{[28]}$ Therefore, the surface modification of nanoparticles with FA increases their accumulation in tumors.

The aims of the present study were the: (1) clustering of SPIONs and modification of the clusters with PEG and FA; (2) enhancement of MRI contrast in tumors by the use of PEGylated SPION nanoclusters (PEG-SPION NCs) and FA-modified PEGylated SPION nanoclusters (FA-PEG-SPION NCs) as contrast agents; (3) clarification of the location of NCs within tumors by histological analyses; (4) evaluation of the therapeutic effect of MHT with intravenous injection of NCs and a safe AC magnetic field; (5) assessment of the hepatotoxicity and nephrotoxicity of NCs.

\section{RESULTS AND DISCUSSION}

We established the method for the one-pot synthesis of allyl-functionalized SPIONs (allyl-SPIONs) via hydrolysis-condensation of a uniquely prepared allyl group-bound iron complex, iron (III) 3-allylacetylacetonate (IAA), at low temperature. ${ }^{[23-25,29]}$ Unlike vinyl groups, allyl groups do not undergo polymerization by heating. Furthermore, they react selectively with thiol groups in high yield of almost $100 \%$ via the thiol-ene click (TEC) reaction regardless of the environment. ${ }^{[29]}$ We achieved the modification of SPIONs with the amino acid cysteine 
in aqueous solution via the TEC reaction between the allyl groups on the surface of SPIONs and the thiol group of cysteine. The clustering of SPIONs and modification of these clusters with PEG and FA were attained simultaneously as follows: (1) allyl-SPIONs were synthesized via the hydrolysis-condensation of IAA; (2) several allyl-SPIONs became enclosed by PEG via the TEC reaction with thiol-functionalized PEG (SH-PEG), resulting in the clustering of SPIONs and PEGylation of the clusters to form PEG-SPION NCs (Figure 1). FA-PEG-SPION NCs were produced when we used thiol- and FA-heterobifunctionalized PEG (SH-PEG-FA) instead of SH-PEG.

Allyl-SPIONs were composed of single-phase magnetite as shown by their X-ray diffraction (XRD) patterns (Supplementary Material: Figure S1). The crystallite size $\left(D_{311}\right)$ was $9.3 \mathrm{~nm}$, as determined using the Scherrer equation. This finding indicated that the crystallinity was relatively high despite the low temperature and short duration of the synthesis.

In the Fourier-transform infrared (FTIR) spectrum of IAA, the absorption bands attributed to the allyl group were observed at $1024 \mathrm{~cm}^{-1}$ and $933 \mathrm{~cm}^{-1}$, and the bands ascribed to $\beta$-diketone were present at $1573 \mathrm{~cm}^{-1}$ and $1459 \mathrm{~cm}^{-1}$ (Supplementary Material: Figure S2). ${ }^{[30]}$ The band due to the $\mathrm{Fe}-\mathrm{O}$ bond of the iron complex was observed at $445 \mathrm{~cm}^{-1}$. These results suggested that IAA was prepared successfully. In the spectrum of allyl-SPIONs, the bands due to the allyl group remained at $1020 \mathrm{~cm}^{-1}$ and $937 \mathrm{~cm}^{-1}$ and those due to $\beta$-diketone also remained $1601 \mathrm{~cm}^{-1}$ and 1455 $\mathrm{cm}^{-1}$. In addition, the bands derived from $v \mathrm{Fe}-\mathrm{O}$ of magnetite appeared at $592 \mathrm{~cm}^{-1}$ and $457 \mathrm{~cm}^{-1}$. These results suggested that allyl-SPIONs were functionalized with allyl groups via the coordinate bond of the $\beta$-diketone. In the spectrum of SH-PEG, the bands attributed to thiol and ether groups were observed at $2512 \mathrm{~cm}^{-1}$ and $1121 \mathrm{~cm}^{-1}$, respectively. In the spectrum of PEG-SPION NCs, the bands derived from thiol and allyl groups disappeared and the bands ascribed to ether, $\beta$-diketone and magnetite remained: the band due to ether was found at $1122 \mathrm{~cm}^{-1}$; the bands due to $\beta$-diketone were found at $1597 \mathrm{~cm}^{-1}$ and $1470 \mathrm{~cm}^{-1}$; and the bands due to magnetite were found at 600 $\mathrm{cm}^{-1}$ and $430 \mathrm{~cm}^{-1}$. These findings demonstrated that allyl-SPIONs reacted successfully with SH-PEG via the TEC reaction and that PEGylation of SPIONs was accomplished. In the spectrum of SH-PEG-FA, the bands attributed to the carboxylic acid of FA, thiol and ether were observed at $1682 \mathrm{~cm}^{-1}, 2516 \mathrm{~cm}^{-1}$ and $1120 \mathrm{~cm}^{-1}$, respectively. In the spectrum of FA-PEG-SPION NCs, the bands derived from thiol and allyl groups disappeared. The bands attributed to carboxylic acid, ether, $\beta$-diketone, and magnetite remained: the band due to carboxylic acid was found at $1656 \mathrm{~cm}^{-1}$; the band due to ether was found at 1118 $\mathrm{cm}^{-1}$; the bands due to $\beta$-diketone were found at 1595 $\mathrm{cm}^{-1}$ and $1465 \mathrm{~cm}^{-1}$; and the bands due to magnetite were found at $608 \mathrm{~cm}^{-1}$ and $421 \mathrm{~cm}^{-1}$. These findings demonstrated that SPIONs were modified successfully with PEG and FA via the TEC reaction between allyl-SPIONs and SH-PEG-FA.

The transmission electron microscopy (TEM) images of PEG-SPION NCs (Supplementary Material: Figure S3) and FA-PEG-SPION NCs (Figure 2) showed that SPIONs with a particle size of 7-9 nm were clustered to have a diameter of $60-100 \mathrm{~nm}$. Furthermore, the clusters were coated with an organic layer, which comprised mainly PEG or FA-PEG, and had a thickness of 4-7 nm. There was little difference in particle size between PEG-SPION NCs and FA-PEG-SPION NCs. The size of PEG-SPION NCs became approximately the same as that of FA-PEG-SPION NCs because the length of the PEG chain of SH-PEG was equal to that of SH-PEG-FA.

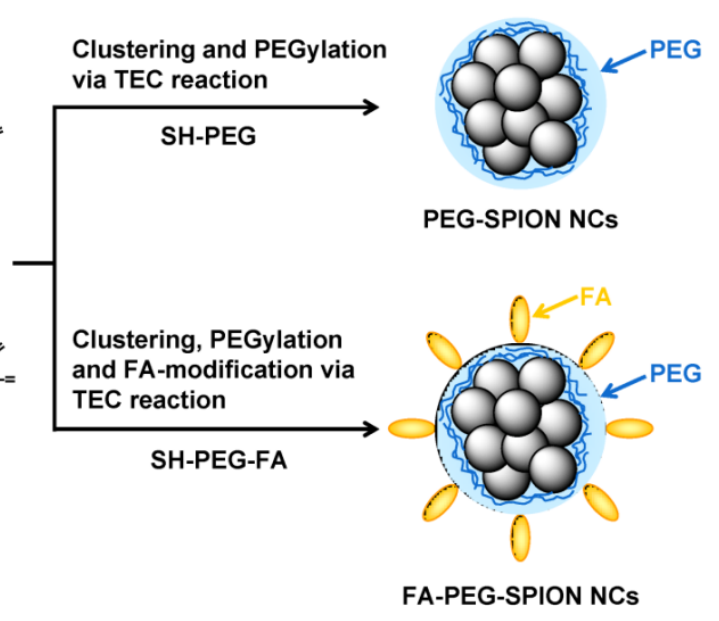

Figure I. Synthesis scheme for PEG-SPION NCs and FA-PEG-SPION NCs. 


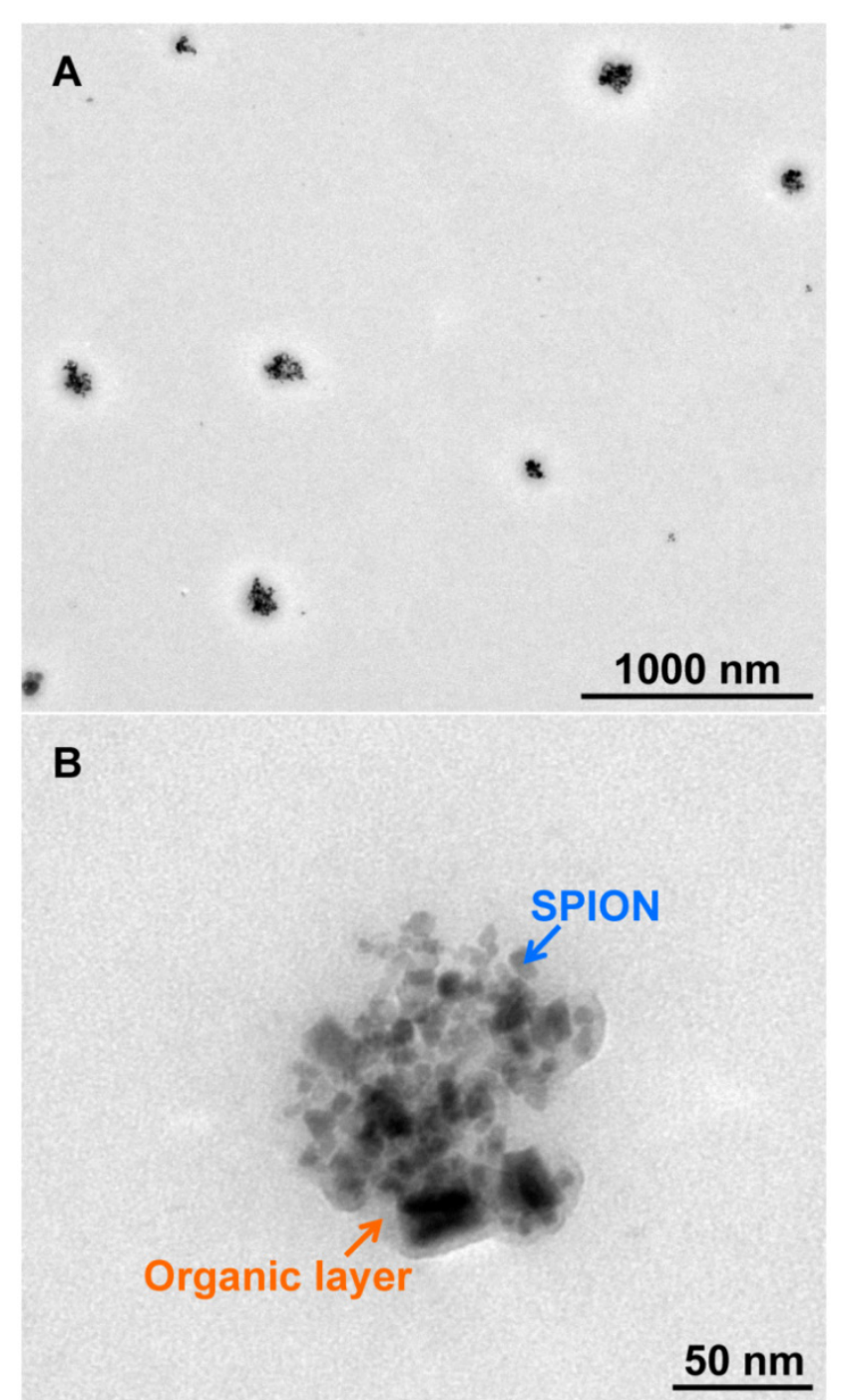

Figure 2. (A) TEM image of FA-PEG-SPION NCs. (B) Close-up image of Figure 2A.

The hydrodynamic diameters $\left(D_{50}\right)$ of allyl-SPIONs, PEG-SPION NCs and FA-PEG-SPION NCs were 8.9, 84.4 and $98.9 \mathrm{~nm}$, respectively, as estimated using dynamic light scattering (DLS) in phosphate-buffered saline (PBS) (Figure 3A). Thus, SPION clustering and surface modification of the clusters created an approximate tenfold increase in the hydrodynamic diameter. Adjustment of the size of the clusters to prevent their leakage from capillaries in normal tissues and making use of the EPR effect would therefore be possible. Moreover, these hydrodynamic diameters agreed with the particle sizes estimated from the TEM images. The $D_{50}$ of FA-PEG-SPION NCs at 3 months after its synthesis was $323 \mathrm{~nm}$ (Supplementary Material: Figure S4), indicating that they gradually formed aggregates over time. However, they were uniformly redispersed in PBS by ultrasonic treatment.
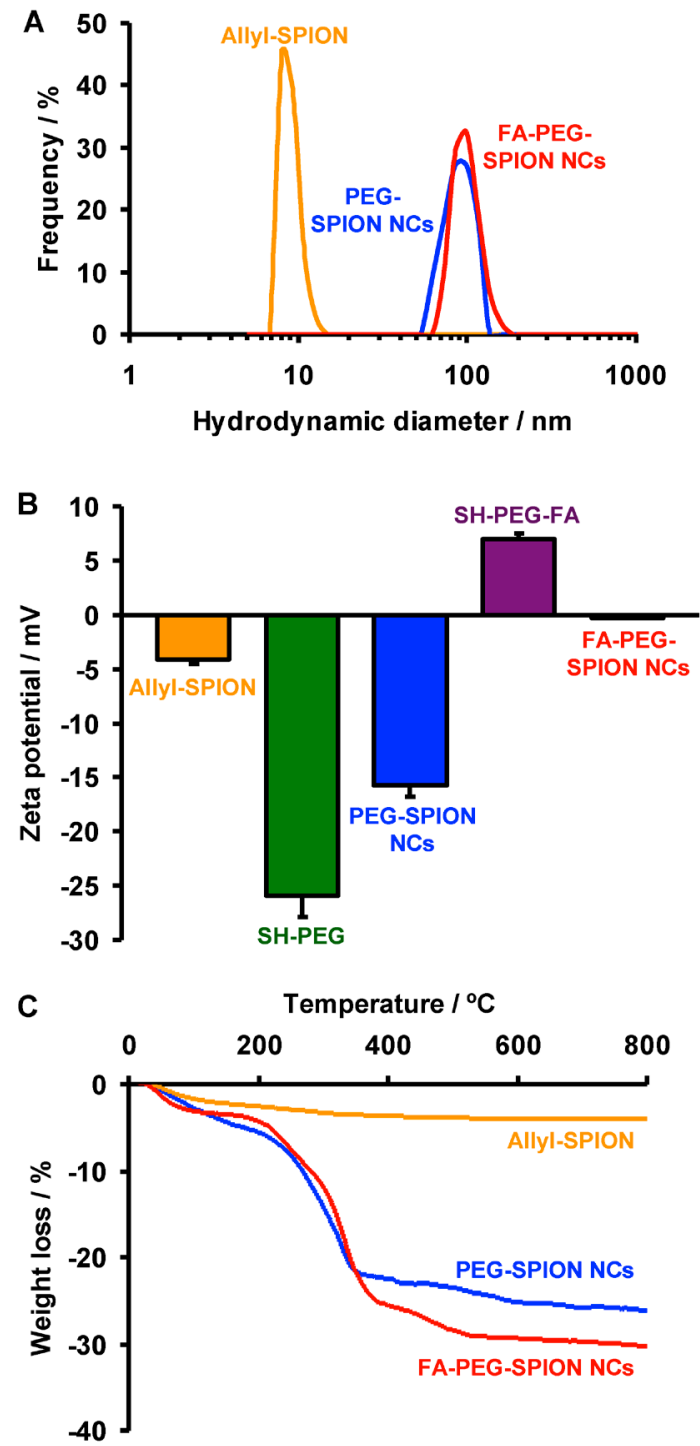

Figure 3. (A) DLS curves of allyl-SPIONs, PEG-SPION NCs and FA-PEG-SPION NCs. (B) Zeta potentials of allyl-SPIONs, SH-PEG, PEG-SPION NCs, SH-PEG-FA and FA-PEG-SPION NCs. (C) TG curves of allyl-SPIONs, PEG-SPION NCs and FA-PEG-SPION NCs.

The zeta potentials of allyl-SPIONs, SH-PEG, PEG-SPION NCs, SH-PEG-FA and FA-PEG-SPION NCs were $-4.2,-25.9,-15.7,7.0$ and $-0.2 \mathrm{mV}$, respectively, in PBS (Figure 3B). The zeta potential of PEG-SPION NCs was lower than that of allyl-SPIONs due to the highly negative charge of PEG, indicating that NCs were covered by PEG. FA-PEG-SPION NCs had a higher zeta potential than allyl-SPIONs because NCs were covered with positively-charged FA-PEG.

The proportions of organic phase in allyl-SPIONs, PEG-SPION NCs and FA-PEG-SPION NCs were 4.0, 26.1 and $29.3 \%$, respectively, as measured by thermogravimetry (TG) (Figure 3C). Thus, the fractions of PEG and FA-PEG in these NCs were estimated to be $22.1 \%$ and $25.3 \%$, respectively. Based on 
these values, the abundances of allyl groups, PEG and FA-PEG were $2.9 \times 10^{-4}, 7.4 \times 10^{-5}$ and $7.4 \times 10^{-5} \mathrm{~mol} / \mathrm{g}$, respectively. Considering that there was little difference in particle size between PEG-SPION NCs and FA-PEG-SPION NCs, as shown in the TEM results, dboth NCs had a nearly equal amount of PEG per not only gram but also particle. Furthermore, a quarter of all allyl groups reacted with SH-PEG or SH-PEG-FA.

The magnetic properties of PEG-SPION NCs, FA-PEG-SPION NCs and the clinical MRI contrast agent Resovist ${ }^{\circledR}$ were measured using a vibrating sample magnetometer (VSM) at room temperature. The magnetization curves of PEG-SPION NCs, FA-PEG-SPION NCs and Resovist showed neither coercivity nor remanent magnetization, indicating that they were superparamagnetic (Supplementary Material: Figure S5). The saturation magnetization $\left(M_{\mathrm{S}}\right)$ of PEG-SPION NCs, FA-PEG-SPION NCs and Resovist were $48.6,44.4$ and $2.4 \mathrm{emu} / \mathrm{g}$, respectively. Thus, PEG-SPION NCs and FA-PEG-SPION NCs had an approximate 20-fold greater $M_{\mathrm{S}}$ than Resovist. Previously, we reported that $8 \mathrm{~nm}$-SPIONs with $28.5 \%$ of organic phase provided $24 \mathrm{emu} / \mathrm{g}$ of $M_{\mathrm{s}}$, which was almost half of the $M_{\mathrm{S}}$ of FA-PEG-SPION NCs. ${ }^{22]}$ This was despite the fact that the $8 \mathrm{~nm}$-SPIONs were synthesized by the same method as used in the present study and had a nearly equal particle size and organic phase to FA-PEG-SPION NCs. These findings demonstrated that the clustering of SPIONs could induce a significant increase in $M_{\mathrm{S}}$ for FA-PEG-SPION NCs, which was consistent with the fact that the clustered magnetic nanoparticles has a much higher magnetic moment than individual magnetic nanoparticles. ${ }^{[16]}$

The relaxivities of PEG-SPION NCs, FA-PEG-SPION NCs and Resovist were measured in agar phantom at 7 T. PEG-SPION NCs and FA-PEG-SPION NCs significantly decreased the MR signal compared with Resovist at an equivalent concentration of iron (Figure 4A). The $r_{2}$ of PEG-SPION NCs and FA-PEG-SPION NCs were markedly higher than that of Resovist (Figure 4B). At the same $r_{2}$, the concentration of PEG-SPION NCs and FA-PEG-SPION NCs were one-tenth of that of Resovist. Thus, PEG-SPION NCs and FA-PEG-SPION NCs had effective relaxivity to ten-times the mass of Resovist. It has been reported that the clustering of SPIONs transfers the size regimes of $r_{2}$ from the motional average regime to the static dephasing regime (SDR) and the value of $r_{2}$ reaches a maximum in the SDR:[14,15] the clusters comprising SPIONs with a diameter of 7-9 $\mathrm{nm}$ have the SDR in 60-110 nm.[14] In PEG-SPION NCs and FA-PEG-SPION NCs, SPIONs with a particle size of 7-9 $\mathrm{nm}$ were clustered to have a diameter of 60-100 nm, as shown in the TEM results. Thus, their cluster sizes were within the SDR. Furthermore, PEG-SPION NCs and FA-PEG-SPION NCs had significantly greater $M_{\mathrm{S}}$ than Resovist, as shown in the VSM results. For these two reasons, the $r_{2}$ of PEG-SPION NCs and FA-PEG-SPION NCs were significantly higher than that of Resovist.

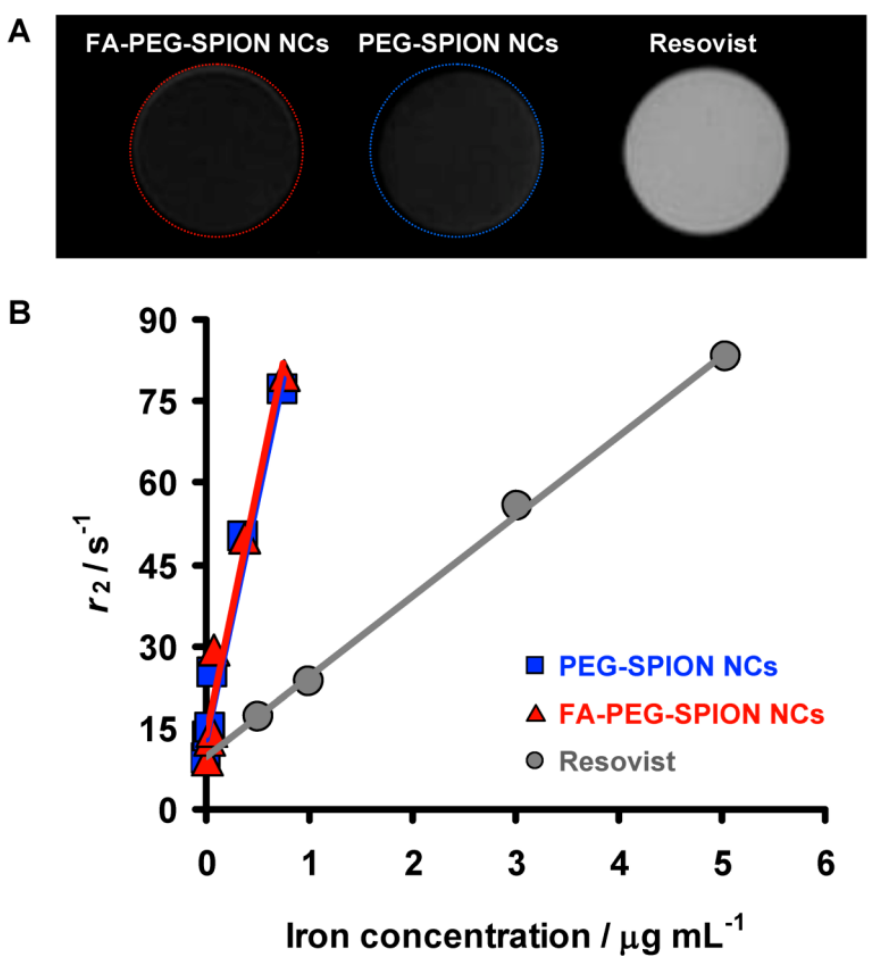

Figure 4. (A) $T_{2}$ MRI of phantoms containing FA-PEG-SPION NCs, PEG-SPION NCs and Resovist. (B) Dependency of the relaxivity of FA-PEG-SPION NCs, PEG-SPION NCs and Resovist upon iron concentration. 
PEG-SPION NCs and FA-PEG-SPION NCs were dissolved in PBS and then injected intravenously into mice bearing subcutaneous xenograft tumors derived from the RPMI8226 multiple myeloma cell line. Twenty-four hours after injection, PEG-SPION NCs and FA-PEG-SPION NCs decreased the MR signal in regions enclosed in the red dot-lined interior of the tumor and heterogeneously enhanced the contrast in the tumor (Figure 5A). In particular, FA-PEG-SPION NCs strongly and extensively enhanced the contrast. By estimating the $T_{2}$ value from the MR images, the $T_{2}$ of the tumor was significantly decreased by injection of PEG-SPION NCs and FA-PEG-SPION NCs, but that of the muscle was unchanged (Figure 5B). In particular, FA-PEG-SPION NCs drastically decreased the $T_{2}$ of the tumor. Furthermore, histological analyses revealed FA-PEG-SPION NCs to be located in cancer tissues (not necrotic tissues) inside the tumor (Figure 5C and Supplementary Material: Figure S6). Hence, only the MRI signal in the cancer tissues was decreased, and that in the necrotic tissues was unchanged, resulting in heterogeneous enhancement of the contrast in the tumor. Thus, the contrast-enhanced regions in the

Figure 5. (A) In vivo $T_{2}$ axial and sagittal maps of mice before and $24 \mathrm{~h}$ after intravenous injection of PEG-SPION NCs and FA-PEG-SPION NCs: $M$ and $T$ indicate muscle and tumor, respectively. (B) Changes in $T_{2}$ values in the tumors and muscles of mice before and $24 \mathrm{~h}$ after intravenous injection of PEG-SPION NCs and FA-PEG-SPION NCs. (C) Histological observation of tumors $24 \mathrm{~h}$ after intravenous injection of FA-PEG-SPION NCs visualized using hematoxylin and eosin staining (left) and Prussian Blue nuclear fast red staining (right): iron is stained blue as indicated by the arrow. (D) Biodistributions of FA-PEG-SPION NCs at $24 \mathrm{~h}$ postinjection. The iron amounts in tissue samples were assessed by nitroso-PSAP $(n=3)$.

B
MRI corresponded to cancer tissues. Moreover, FA-PEG-SPION NCs occupied $\approx 12 \%$ of the area of the cancer tissues in this histological image according to the software (Image-Pro plus). In addition, FA-PEG-SPION NCs were somewhat present in the liver and spleen but were not observed in the heart, lung and kidney (Supplementary Material: Figure S7).
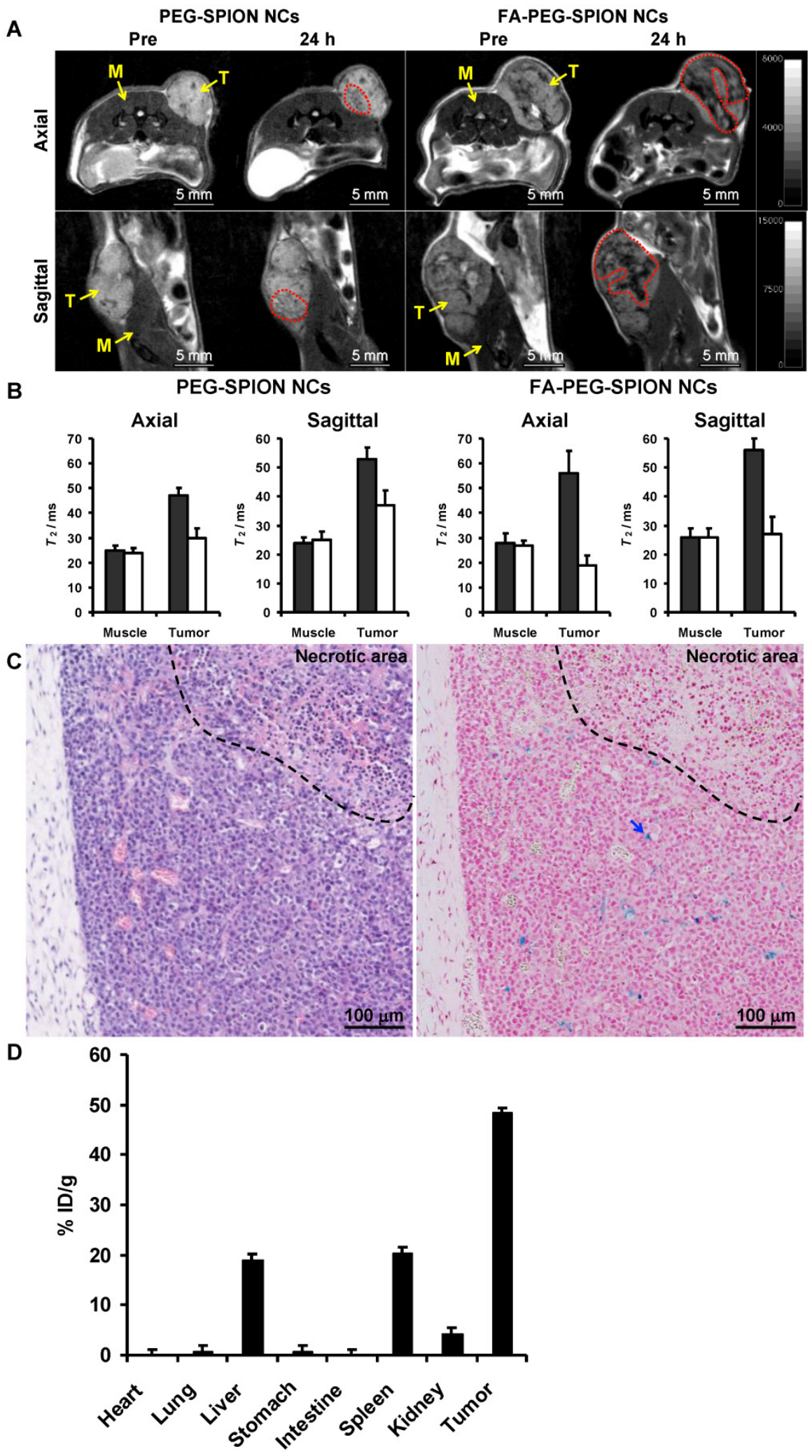
The biodistribution of FA-PEG-SPION NCs in major organs including tumor was assessed by nitroso-PSAP method at $24 \mathrm{~h}$ postinjection. As shown in Figure 5D, the tumor had the highest uptake followed by the spleen, liver and kidney. Approximately 50\% injected dose/gram of tissue (\% ID/g) was found in the tumor, and the concentration of FA-PEG-SPION $\mathrm{NCs}$ in the tumor was $24 \mathrm{nmol} \mathrm{Fe} / \mathrm{g}$. Whereas nanoparticles generally accumulate in the liver and spleen because of reticuloendothelial system (RES) uptake, FA-PEG-SPION NCs were likely to accumulate in the tumor rather than the RES. Few FA-PEG-SPION NCs accumulated in the heart, lung, stomach and intestine. Furthermore, about $6 \% \mathrm{ID} / \mathrm{g}$ was not found in the above organs, suggesting that FA-PEG-SPION NCs were excreted in the urine or feces.

FA-PEG-SPION NCs were hydrophilic and dispersed uniformly in water (Figure 6A). They generated heat by application of an AC magnetic field with $H=8 \mathrm{kA} / \mathrm{m}$ and $f=230 \mathrm{kHz}\left(H f=1.8 \times 10^{9} \mathrm{~A} / \mathrm{m} \cdot \mathrm{s}\right)$ (Figure 6B) and they increased the temperature of the solution by $\approx 20^{\circ} \mathrm{C}$ for $10 \mathrm{~min}$ (Figure 6C). Similarly, allyl-SPIONs also increased the temperature of the solution by $\approx 18^{\circ} \mathrm{C}$ for $10 \mathrm{~min}$ at the same concentration level. The specific absorption rate (SAR) was calculated using the following equation:

$$
\text { SAR }=\left(C_{w} / m\right)(d T / d t),
$$

where $C_{\mathrm{w}}$ is the specific heat of water and $m$ is the concentration of SPIONs in solution. The average SAR values of FA-PEG-SPION NCs and allyl-SPIONs were 248 and $156 \mathrm{~W} / \mathrm{g}_{\mathrm{Fe}}$, respectively. In addition, we previously revealed that 8-nm SPIONs also had a SAR of $156 \mathrm{~W} / \mathrm{g}_{\mathrm{Fe}} .{ }^{[29]}$ Thus, FA-PEG-SPIONs provided a 1.6-fold greater SAR than non-clustered SPIONs. These findings demonstrated that SPION clustering could induce an increase in not only $r_{2}$ but also the SAR.

FA-PEG-SPION NCs were dissolved in PBS, and then injected intravenously into tumor-bearing mice. Twenty-four hours after injection, the mice were placed in an AC magnetic field with $H=8 \mathrm{kA} / \mathrm{m}$ and $f$ $=230 \mathrm{kHz}\left(H f=1.8 \times 10^{9} \mathrm{~A} / \mathrm{m} \cdot \mathrm{s}\right)$ for $20 \mathrm{~min}$. The groups $(n=5)$ were as follows: mice without treatment; mice injected intravenously with FA-PEG-SPION NCs; mice placed in an AC magnetic field for $20 \mathrm{~min}$; mice placed in an AC magnetic field for $20 \mathrm{~min}$ the day after intravenous injection of FA-PEG-SPION NCs. The tumors of mice injected with FA-PEG-SPION NCs underwent local heating by application of an AC magnetic field (Figure 7A). The temperature of the tumor was higher than the surrounding tissues by $\approx 6^{\circ} \mathrm{C}$ at $20 \mathrm{~min}$ after treatment. Based on the temperature-time curve of the tumor (Supplementary Material: Figure S8), the SAR of
FA-PEG-SPION NCs in the tumor was calculated using the following equation:

$$
\operatorname{SAR}=\left(C_{\mathrm{t}} / m_{\mathrm{t}}\right)(\mathrm{d} T / \mathrm{d} t),
$$

where $C_{\mathrm{t}}$ is the specific heat of tissue $(\approx 3.5 \mathrm{~J} / \mathrm{g} \cdot \mathrm{K}$ at $\left.37^{\circ} \mathrm{C}\right)^{[31]}$ and $m_{\mathrm{t}}$ is the concentration of SPIONs in the tumor. The average SAR was $104 \mathrm{~W} / \mathrm{g}_{\mathrm{Fe}}$, which was lower than the SAR in water. This may be because of the thermal diffusion caused by blood flow. As shown in Figure 7B, tumor growth was significantly inhibited in mice placed in an AC magnetic field after injection of FA-PEG-SPION NCs. There was little difference in the growth behavior of tumors between control mice. Thirty-five days after treatment, the tumor volume of mice placed in an AC magnetic field after injection of FA-PEG-SPION NCs was one-tenth that of the control mice. The photographs of mice 35 days after treatment also demonstrated that mice placed in an AC magnetic field after injection of FA-PEG-SPION NCs had appreciably smaller tumors than control mice (Figure 7D). Furthermore, mice placed in an AC magnetic field after injection of FA-PEG-SPION NCs were alive after 12 weeks; control mice died up to 8 weeks after treatment (Figure 7C). These results demonstrated that MHT involving intravenous injection of FA-PEG-SPION NCs and application of a safe AC magnetic field inhibited tumor growth and improved survival. MHT could be a new treatment for multiple myeloma, which is hard to cure because the cancer cells have marked resistance to chemotherapeutic agents. ${ }^{[32]}$
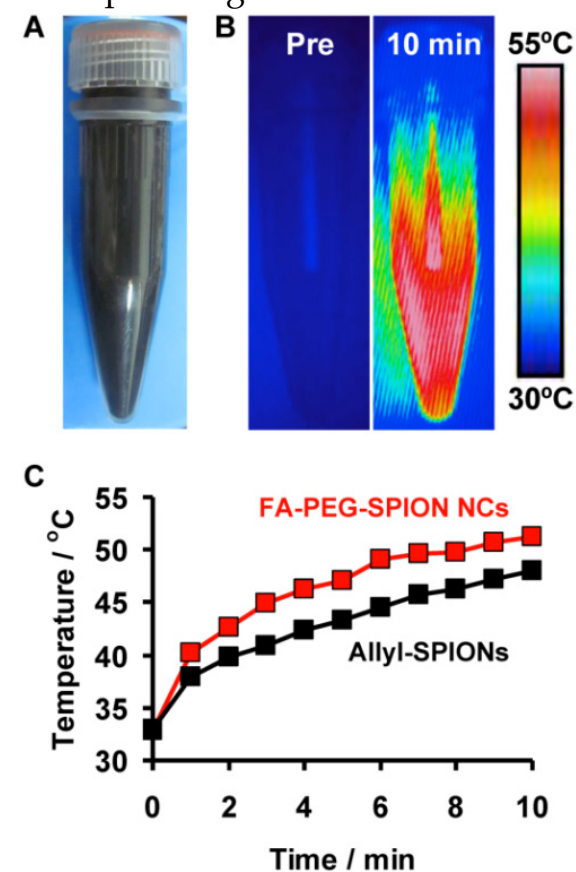

Figure 6. (A) Photograph of FA-PEG-SPION NCs in water. (B) Thermal images of FA-PEG-SPION NCs in water before and $10 \mathrm{~min}$ after application of an $\mathrm{AC}$ magnetic field with $H=8 \mathrm{kA} / \mathrm{m}$ and $f=230 \mathrm{kHz}$. (C) Temperature-time curves of FA-PEG-SPION NCs and allyl-SPIONs in water under the $A C$ magnetic field. 

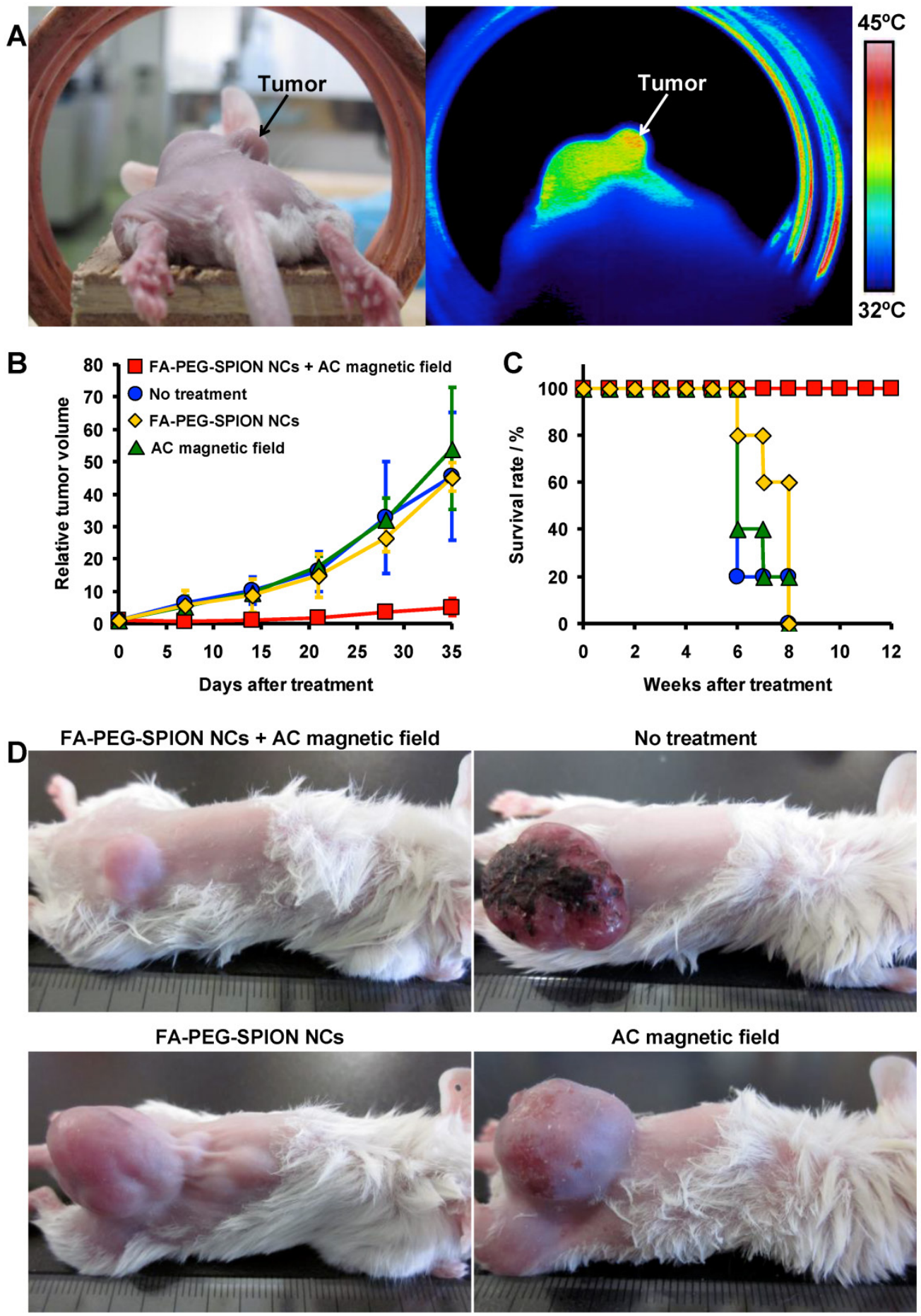

Figure 7. (A) Photograph (left) and thermal image (right) of a mouse $24 \mathrm{~h}$ after intravenous injection of FA-PEG-SPION NCs under an AC magnetic field with $H=8 \mathrm{kA} / \mathrm{m}$ and $f=230 \mathrm{kHz}$. (B) Tumor-growth behavior and (C) survival period of mice without treatment and treated by intravenous injection of FA-PEG-SPION NCs, application of an AC magnetic field, and application of an AC magnetic field $24 \mathrm{~h}$ after intravenous injection of FA-PEG-SPION NCs $(n=5)$. (D) Photographs of mice 35 days after treatment.

The hepatotoxicity and nephrotoxicity of FA-PEG-SPION NCs were evaluated using biochemical assays in the sera of mice 1 month after injection. There was little difference between the levels of blood urea nitrogen (BUN), alkaline phosphatase (ALP), alanine aminotransferase (ALT), and aspartate ami- notransferase (AST) in the sera of mice injected with FA-PEG-SPION NCs, and those in the sera of mice injected with PBS (Figure 8). These results suggested that FA-PEG-SPION NCs were neither hepatotoxic nor nephrotoxic. 


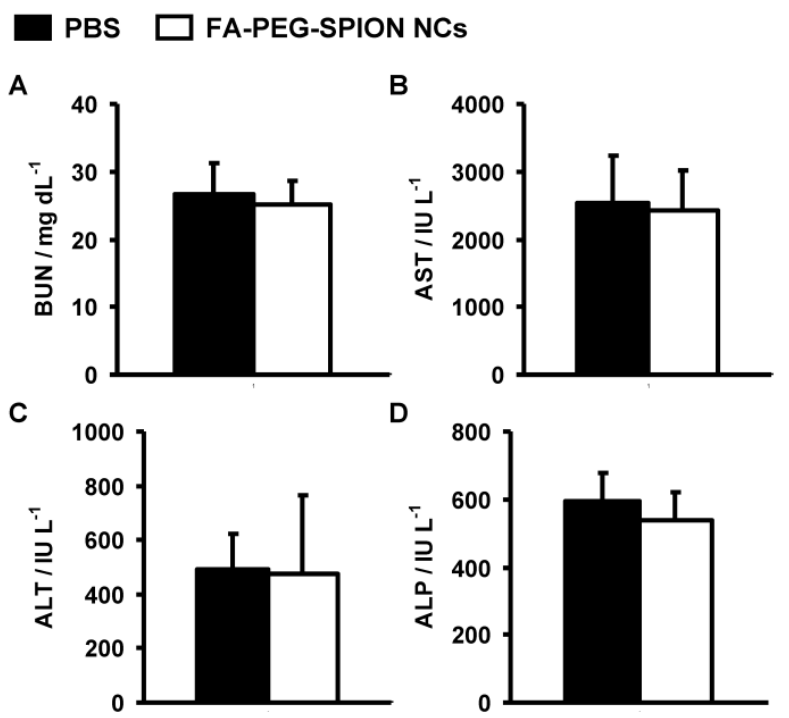

Figure 8. Biochemical assays in the sera of mice injected with PBS and FA-PEG-SPION NCs. One month after injection, (A) BUN, (B) AST, (C) ALT, and (D) ALP values were measured.

\section{CONCLUSION}

In summary, the TEC reaction of allyl-SPIONs with SH-PEG or SH-PEG-FA led to SPION clustering and modification of PEG or FA-PEG to produce PEG-SPION NCs and FA-PEG-SPION NCs. SPION clustering could increase the $M_{\mathrm{S}}$ of these NCs, resulting in an increase in $r_{2}$ and the SAR. PEG-SPION NCs and FA-PEG-SPION NCs provided higher relaxivity than Resovist. Twenty-four hours after intravenous injection of PEG-SPION NCs and FA-PEG-SPION NCs into mice bearing tumors derived from a multiple myeloma cell line, NCs accumulated locally in cancer (not necrotic) tissues within the tumor. As a result, in the cancer tissues, $T_{2}$ was significantly decreased and contrast enhanced. In particular, FA-PEG-SPION NCs strongly and extensively enhanced the contrast of cancer tissues. Furthermore, application of a safe AC magnetic field with $H=8$ $\mathrm{kA} / \mathrm{m}$ and $f=230 \mathrm{kHz}\left(H f=1.8 \times 10^{9} \mathrm{~A} / \mathrm{m} \cdot \mathrm{s}\right)$ to tumor-bearing mice the day after intravenous administration of FA-PEG-SPION NCs significantly inhibited tumor growth. In addition, FA-PEG-SPION NCs were neither hepatotoxic nor nephrotoxic. Multiple myeloma remains an incurable malignancy because of marked resistance of cancer cells to chemotherapeutic agents. FA-PEG-SPION NCs could be used to enable the detection of multiple myeloma using MRI, and the growth of multiple myeloma could be inhibited using MHT.

\section{METHODS}

\section{Chemicals}

IAA was prepared by the method described in the literature. ${ }^{[33]}$ Hydrazine was purchased from Tokyo Chemical Industry (Tokyo, Japan). 2,2'-azobis[2-(2-imidazolin-2-yl)propane]dihydrochlo ride (AIPD) was obtained from Wako Pure Chemicals (Osaka, Japan). SH-PEG $\left(M_{W}=3000\right)$ was purchased from NOF (Tokyo, Japan). SH-PEG-FA $\left(M_{W}=3000\right)$ was from Nanocs (NY, USA).

\section{Synthesis of AllyI-SPIONs}

Synthesis was carried out according to a previously reported procedure. ${ }^{[23-25,29,34]}$ Briefly, $35 \mu \mathrm{mol}$ of hydrazine and $102 \mu \mathrm{mol}$ of water were added to an ethanolic solution of IAA $(7.0 \mathrm{mM})$. The solution was heated at $80^{\circ} \mathrm{C}$ for $2 \mathrm{~h}$ and then centrifuged at $20,000 \times \mathrm{g}$ for $10 \mathrm{~min}$ to precipitate allyl-SPIONs. The separated allyl-SPIONs were washed thrice with water and dispersed in water.

\section{Formation of PEG-SPION NCs and FA-PEG-SPION NCs by the TEC Reaction}

PEG-SPION NCs and FA-PEG-SPION NCs were produced by making improvements to the methods described in our previous study. ${ }^{[29]}$ AIPD $(26 \mu \mathrm{mol})$ and $1.7 \mu \mathrm{mol}$ of SH-PEG or SH-PEG-FA were added to an aqueous solution of allyl-SPIONs. The solution was heated at $60^{\circ} \mathrm{C}$ for $3 \mathrm{~h}$ and then centrifuged at 20,000 $\times \mathrm{g}$ for $10 \mathrm{~min}$ to precipitate PEG-SPION NCs and FA-PEG-SPION NCs. PEG-SPION NCs and FA-PEG-SPION NCs were washed thrice with water and dispersed in PBS.

\section{Characterization}

The size and shape of FA-PEG-SPION NCs were observed by a transmission electron microscope (H-760, Hitachi, Tokyo, Japan). The hydrodynamic diameter and zeta potential were measured by DLS (NICOMP 380 ZLS, Showa Denko, Tokyo, Japan). FTIR spectra were recorded with a FTIR spectrometer (Nexus 470, Nicolet, Madison, WI, USA). The amount of organic phase were measured using TG (TG8120, Rigaku, Tokyo, Japan) at a heating rate of $10^{\circ} \mathrm{C} / \mathrm{min}$ from room temperature to $800^{\circ} \mathrm{C}$ under an oxygen atmosphere. The crystalline phases were analyzed by XRD using $\mathrm{Cu} \mathrm{Ka}$ radiation with a monochromator (RINT-2500, Rigaku, Tokyo, Japan). Magnetic properties were measured with a vibrating sample magnetometer (Type 5, Toei Kogyo, Tokyo, Japan) at room temperature. 


\section{Ethics Statement}

The study protocol was approved by the Animal Care and Use Committee of the University of Tokushima (Tokushima, Japan).

\section{Animals}

Female CB17/Icr-Prkdscid mice aged 4 weeks were purchased from Charles River Laboratories (Yokohama, Japan) and maintained in a specific pathogen-free facility in our Animal Resources Center. To eradicate residual natural killer (NK) cells, mice were injected intraperitoneally with $10 \mu \mathrm{L}$ of rabbit anti-asialo GM1 antiserum (Wako Pure Chemicals) 1 day before tumor inoculation. ${ }^{[35]}$ Inoculation with human multiple myeloma cells $\left(5 \times 10^{6}\right)$ was accomplished by subcutaneous injection into the backs of mice.

\section{MRI}

PEG-SPION NCs or FA-PEG-SPION NCs were dispersed homogeneously in phantom agar, which was composed of $1 \mathrm{wt} \%$ agar and $99 \mathrm{wt} \%$ water. MRIs of the phantom agar were taken using a 7.0 T Bruker Biospec 70/20 USR nuclear magnetic resonance spectrometer (Ettlingen, Germany). MRIs of mice $(n=3$ per cohort) were acquired before and $24 \mathrm{~h}$ after intravenous injection of PEG-SPION NCs and FA-PEG-SPION NCs $(48 \mu \mathrm{mol} \mathrm{Fe} / \mathrm{kg})$. After anesthetizing the mice with a mixture of oxygen and isoflurane, sagittal and axial images were recorded using a Bruker BGA-S coil. A $T_{2}$ mapping sequence used was a multi-slice multi-echo (MSME) sequence, and the parameters were set as follows: $\mathrm{TR}=2500.0 \mathrm{~ms}$, $\mathrm{TE}=11.0,22.0,33.0,44.0,55.0$ and $66.0 \mathrm{~ms}$; number of average 2; scan time $16 \mathrm{~min} ; \mathrm{FA}=180.0^{\circ}$; slice thickness $=1.00 \mathrm{~mm}$; FOV $=3 \times 3 \mathrm{~cm}$; slices 7 , matrix $256 \times 256$; pixel size $117 \times 117 \mu \mathrm{m}$; motion suppression on. All images were processed with ParaVision software (Bruker Biospin). T2 was calculated from the t2vtr-fitting function (Bruker Biospin).

\section{Hyperthermia Experiments}

All hyperthermia experiments were carried out safely using an applied AC field of $8 \mathrm{kA} / \mathrm{m}$ and a frequency of $230 \mathrm{kHz}\left(\mathrm{H} f=1.8 \times 10^{9} \mathrm{~A} / \mathrm{m} \cdot \mathrm{s}\right)$. An AC magnetic field was generated using a transistor inverter with field coils $(2.4 \mathrm{~kW}$, EasyHeat, Ambrell, NY, USA). Thermal images were taken using a Thermograph (Thermo Gear G100EX, NEC Avio Infrared Technologies, Tokyo, Japan), as were temperature measurements. The SAR values of FA-PEG-SPION NCs and allyl-SPIONs were evaluated using their aqueous solution $(2.2 \mathrm{mg}$ sPION $/ \mathrm{mL})$. In vivo hyperthermia experiments were carried out on mice with tumors of diameter $50 \mathrm{~mm}^{3}$. Tumor volumes were calculated from the formula

$$
\mathrm{V}=\mathrm{AB}^{2} \Pi / 6,
$$

where $\mathrm{A}$ is the longer and $\mathrm{B}$ is the shorter lateral diameter of the tumor. Mice were divided into four groups ( $n=5$ per cohort): no treatment; intravenous administration of FA-PEG-SPION NCs (48 $\mu \mathrm{mol}$ $\mathrm{Fe} / \mathrm{kg})$; application of an AC magnetic field for 20 min; and $24 \mathrm{~h}$ after intravenous administration of FA-PEG-SPION NCs $(48 \mu \mathrm{mol} \mathrm{Fe} / \mathrm{kg})$, application of an AC magnetic field for $20 \mathrm{~min}$. Treatments were carried out once a day as soon as the tumor reached a diameter of $50 \mathrm{~mm}^{3}$. Mice were killed when the tumor reached a diameter of $3,500 \mathrm{~mm}^{3}$.

\section{Histological Analyses}

Tissue samples obtained for histology were immediately immersed in $4 \%$ paraformaldehyde solution for $24 \mathrm{~h}$. Fixed tissues were encased in paraffin blocks. Tissue sections $(2 \mu \mathrm{m})$ were cut from paraffin blocks and affixed to Superfrost Microscopy Slides. Prussian Blue staining is a highly sensitive method for identifying ferric species, and was used with a nuclear fast red counterstain to visualize FA-PEG-SPION NC content in the tissue sections. Briefly, microscopy slides containing tissue sections were sequentially immersed in xylene to dissolve excess paraffin and rehydrated through immersion in decreasing concentrations $(100-50 \%)$ of ethanol in water followed by $100 \%$ water. Slides were then immersed in a freshly prepared solution containing equal parts $2 \% \mathrm{HCl}$ and $2 \%$ potassium ferrocyanide for $15 \mathrm{~min}$. Prussian Blue-stained slides were rinsed in water and immersed in nuclear fast red solution for $5 \mathrm{~min}$. Slides were then rinsed in water, dehydrated through increasing concentrations (50-100\%) of ethanol, and cleared in xylene. Dehydrated tissues were coverslipped with Permount resinous mounting medium and allowed to dry overnight at ambient temperature. Stained slides were observed under light microscopy. Hematoxylin and eosin staining of tissue sections was carried out in a conventional manner.

\section{Biodistribution}

The tumor-bearing mice were injected intravenously with FA-PEG-SPION NCs $(48 \mu \mathrm{mol} \mathrm{Fe} / \mathrm{kg})$ and PBS. At $24 \mathrm{~h}$ postinjection, animals were sacrificed, and the tissues were taken out, washed, and then weighed. The tissue sample $(10 \mathrm{mg})$ was dissolved with $1 \mathrm{~mL}$ of aqua regia in a Teflon vials. The tissue solution $(0.1 \mathrm{~mL})$ was diluted with $1.5 \mathrm{~mL}$ of ultrapure water. The iron concentration in the tissue solution was measured by nitroso-PSAP method us- 
ing an iron assay kit (Metallogenics ${ }^{\mathrm{TM}}, \mathrm{AKJ}$ Global Technology Co., Ltd., Chiba, Japan) according to the manufacturer's instructions. Data presented in this work were obtained from averaging three animals.

\section{Toxicity Evaluation}

PBS $(100 \mu \mathrm{L})$ and the FA-PEG-SPION NCs $(48$ $\mu \mathrm{mol} \mathrm{Fe} / \mathrm{kg}$ ) were injected intravenously into mice ( $n$ $=5$ per cohort). One month after injection, blood samples were collected, and serum was obtained by centrifugation of whole blood at 3,000 rpm for $15 \mathrm{~min}$. Liver function was evaluated based on the serum levels of ALP, ALT and AST. Nephrotoxicity was determined by BUN. These biochemical parameters were determined using an automated biochemical analyzer (Hitachi 7180, Tokyo, Japan).

\section{Supplementary Material}

Fig.S1 - S8. http://www.thno.org/v03p0366s1.pdf

\section{Acknowledgements}

This work was supported in part by Grant-in-Aid for Young Scientists (B) (24760551) from the Ministry of Education, Science, Sports, and Culture of Japan.

\section{Competing Interests}

The authors have declared that no competing interest exists.

\section{References}

1. Bardhan R, Lal S, Joshi A, Halas NJ. Theranostic nanoshells: from probe design to imaging and treatment of cancer. Accounts Chem Res. 2011; 44: 936-46.

2. Janib SM, Moses AS, MacKay JA. Imaging and drug delivery using theranostic nanoparticles. Adv Drug Deliv Rev. 2010; 62: 1052-63.

3. Barreto JA, O'Malley W, Kubeil M, Graham B, Stephan H, Spiccia L. Nanomaterials: applications in cancer imaging and therapy. Adv Mater. 2011; 23: H18-40.

4. Kelkar SS, Reineke TM. Theranostics: combining imaging and therapy. Bioconjugate Chem. 2011; 22: 1879-903.

5. Doane TL, Burda C. The unique role of nanoparticles in nanomedicine: imaging, drug delivery and therapy. Chem Soc Rev. 2012; 41: 2885-911.

6. Laurent S, Forge D, Port M, Roch A, Robic C, Elst LV, Muller RN. Magnetic iron oxide nanoparticles: synthesis, stabilization, vectorization, physicochemical characterizations, and biological applications. Chem Rev. 2008; 108: 2064-110.

7. Mornet S, Vasseur S, Grasset F, Duguet E. Magnetic nanoparticle design for medical diagnosis and therapy. J Mater Chem. 2004; 14: 2161-75.

8. Cole AJ, Yang VC, David AE. Cancer theranostics: the rise of targeted magnetic nanoparticles. Trends Biotechnol. 2011; 29: 323-32.

9. Xie J, Jon S. Magnetic nanoparticle-based theranostics. Theranostics. 2012; 2: $122-4$.

10. Kessinger CW, Togao O, Khemtong C, Huang G, Takahashi M, Gao J. investigation of in vivo targeting kinetics of $\alpha_{v} \beta_{3}$-specific superparamagnetic nanoprobes by time-resolved MRI. Theranostics. 2011; 1: 263-73.

11. Li M, Kim HS, Tian L, Yu MK, Jon S, Moon WK. Comparison of two ultrasmall superparamagnetic iron oxides on cytotoxicity and MR imaging of tumors. Theranostics. 2012; 2: 76-85.

12. Huang J, Zhong $X$, Wang L, Yang L, Mao H. Improving the magnetic resonance imaging contrast and detection methods with engineered magnetic nanoparticles. Theranostics 2012; 2: 86-102.
13. Shao H, Min C, Issadore D, Liong M, Yoon TJ, Weissleder R, Lee H. Magnetic nanoparticles and microNMR for diagnostic applications. Theranostics. 2012; 2: 55-65.

14. Pöselt E, Kloust H, Tromsdorf U, Janschel M, Hahn C, Maßlo C, Weller H. Relaxivity optimization of a PEGylated iron-oxide-based negative magnetic resonance contrast agent for $\mathrm{T}_{2}$-weighted spin echo imaging. ACS Nano. 2012; 6: 1619-24.

15. Min C, Shao H, Liong M, Yoon TJ, Weissleder R, Lee H. Mechanism of magnetic relaxation switching sensing. ACS Nano. 2012; 6: 6821-8.

16. Qiu P, Jensen C, Charity N, Towner R, Mao C. Oil phase evaporation-induced self-assembly of hydrophobic nanoparticles into spherical clusters with controlled surface chemistry in an oil-in-water dispersion and comparison of behaviors of individual and clustered iron oxide nanoparticles. J Am Chem Soc. 2010; 132: 17724-32.

17. Laurent S, Dutz S, Häfeli UO, Mahmoudi M. Magnetic fluid hyperthermia: focus on superparamagnetic iron oxide nanoparticles. Adv Colloid Interface Sci. 2011; 166: 8-23.

18. Alphandéry E, Faure S, Seksek O, Guyot F, Chebbi I. Chains of magnetosomes extracted from AMB-1 magnetotactic bacteria for application in alternative magnetic field cancer therapy. ACS Nano. 2011; 5: 6279-96.

19. Lee JH, Jang Jt, Choi Js, Moon SH, Noh Sh, Kim Jw, Kim JG, Kim IS, Park KI, Cheon J. Exchange-coupled magnetic nanoparticles for efficient heat induction. Nat Nanotechnol. 2011; 6: 418-22.

20. Zhao Q, Wang L, Cheng R, Mao L, Arnold RD, Howerth EW, Chen ZG, Platt S. Magnetic nanoparticle-based hyperthermia for head \& neck cancer in mouse models. Theranostics. 2012; 2: 113-21.

21. Guardia P, Corato RD, Lartigue L, Wilhelm C, Espinosa A, Garcia-Hernandez M, Gazeau F, Manna L, Pellegrino T. Water-soluble iron oxide nanocubes with high values of specific absorption rate for cancer cell hyperthermia treatment. ACS Nano. 2012; 6: 3080-91.

22. Hergt R, Dutz S. Magnetic particle hyperthermia-biophysical limitations of a visionary tumour therapy. J Magn Magn Mater. 2007; 311: 187-92.

23. Hayashi K, Moriya M, Sakamoto W, Yogo T. Chemoselective synthesis of folic acid-functionalized magnetite nanoparticles via click chemistry for magnetic hyperthermia. Chem Mater. 2009; 21: 1318-25.

24. Hayashi K, Ono K, Suzuki H, Sawada M, Moriya M, Sakamoto W, Yogo T. High-frequency, magnetic-field-responsive drug release from magnetic nanoparticle/organic hybrid based on hyperthermic effect. ACS Appl Mater Interfaces. 2010; 2: 1903-11.

25. Hayashi K, Shimizu Y, Asano H, Sakamoto W, Yogo T. Synthesis of spinel iron oxide nanoparticle/organic hybrid for hyperthermia. J Mater Res. 2008; 23: 3415-24.

26. Maeda H, Wu J, Sawa T, Matsumura Y, Hori K. Tumor vascular permeability and the EPR effect in macromolecular therapeutics: a review. J Control Release. 2000; 65: 271-84.

27. Harris JM, Chess RB. Effect of PEGylation on pharmaceuticals. Nat Rev Drug Discov. 2003; 2: 214-21.

28. Yu MK, Park J, Jon S. Targeting strategies for multifunctional nanoparticles in cancer imaging and therapy. Theranostics. 2012; 2: 3-44.

29. Hayashi K, Ono K, Suzuki H, Sawada M, Moriya M, Sakamoto W, Yogo T. One-pot biofunctionalization of magnetic nanoparticles via thiol-ene click reaction for magnetic hyperthermia and magnetic resonance imaging. Chem Mater. 2010; 22: 3768-72.

30. Silverstein RM, Webster FX, Kiemle DJ. Spectrometric identification of organic compounds, 7th Ed. New York, USA: John Wiley \& Sons; 2005.

31. Haemmerich D, Santos Id, Schutt DJ, Webster JG, Mahvi DM. In vitro measurements of temperature-dependent specific heat of liver tissue. Med Eng Phys. 2006; 28: 194-7.

32. Alexanian R, Dimopoulos M. The treatment of multiple myeloma. New Engl J Med. 1994; 330: 484-9.

33. Tayim TA, Sabri M. Synthesis of some olefin-substituted metal acetylacetonates. Inorg Nucl Chem Lett. 1973; 9: 753-7.

34. Hayashi K, Ono K, Suzuki H, Sawada M, Moriya M, Sakamoto W, Yogo T. Electrosprayed synthesis of red-blood-cell-like particles with dual modality for magnetic resonance and fluorescence imaging. Small. 2010; 6: 2384-91.

35. Ozaki S, Kosaka M, Wakatsuki S, Abe M, Koishihara Y, Matsumoto T. Immunotherapy of multiple myeloma with a monoclonal antibody directed against a plasma cell-specific antigen, HM1.24. Blood. 1997; 90: 3179-86. 